\title{
Berezinskii-Kosterlitz-Thouless transition transport in spin-triplet superconductor
}

\author{
Suk Bum Chung ${ }^{1,2,3 \star}$ and Se Kwon Kim ${ }^{4}$ \\ 1 Department of Physics and Natural Science Research Institute, \\ University of Seoul, Seoul 02504, Republic of Korea \\ 2 School of Physics, Korea Institute for Advanced Study, Seoul 02455, Republic of Korea \\ 3 Center for Correlated Electron Systems, Institute for Basic Science (IBS), \\ Seoul National University, Seoul 08826, Republic of Korea \\ 4 Department of Physics, KAIST, Daejeon 34141, Republic of Korea \\ $\star$ sbchung0@uos.ac.kr
}

\begin{abstract}
As the spin-triplet superconductivity arises from the condensation of spinful Cooper pairs, its full characterization requires not only charge ordering, but also spin ordering. For a two-dimensional (2D) easy-plane spin-triplet superconductor, this naïvely seems to suggest the possibility of two distinct Berezinskii-Kosterlitz-Thouless (BKT) phase transitions, one in the charge sector and the other in the spin sector. However, it has been recognized that there are actually three possible BKT transitions, involving the deconfinement of, respectively, the conventional vortices, the merons and the half-quantum vortices with vorticity in both the charge and the spin current. By considering equalspin-pairing spin-triplet superconductors with bulk spin degeneracy, we show how all the transitions can be characterized by the relation between the voltage drop and the spin-polarized current bias. This study reveals that, due to the hitherto unexamined transport of half-quantum vortices, there is an upper bound on the spin supercurrent in a quasi-long range ordered spin-triplet superconductor, which provides a means for half-quantum vortex detection via transport measurements and deeper understanding of fluctuation effects in superconductor-based spintronic devices.
\end{abstract}

(c) $\underset{\substack{\text { gr } \\ \text { (1) }}}{ }$ Copyright S. B. Chung and S. K. Kim This work is licensed under the Creative Commons Attribution 4.0 International License.

Published by the SciPost Foundation.
Received 07-07-2021

Accepted 09-11-2021

Published 27-01-2022

doi:10.21468/SciPostPhysCore.5.1.003

\section{Contents}

1 Introduction

2 General considerations

3 Transport signature of BKT transitions

3.1 Transport setup

3.2 Full-quantum vortex deconfinement

$3.3 d$-vector meron deconfinement 
3.4 Half-quantum vortex deconfinement

4 Conclusion

A The derivation of Eq. (5)

B $\Delta V$ near the $d$-vector meron deconfinement

C $\Delta V$ near the half-quantum vortex deconfinement

\section{Introduction}

One defining feature of the spin-triplet superconducting phase is the concurrent breaking of spin-rotational symmetry and gauge symmetry [1-4]. Recent years have seen newer candidates for spin-triplet superconductors in uranium-based materials [5-9], doped topological insulator [10,11], spin-orbit-coupled materials [12-14], and magic angle graphene [15-20], notwithstanding controversies concerning older candidate $\mathrm{Sr}_{2} \mathrm{RuO}_{4}[3,21,22]$. It has been recognized that the spatial variation of the Cooper pair spin state would lead to spin current carried by Cooper pairs [23-25]. In other words, spin-triplet superconductor can support superfluid spin transport, a spin analogue of mass transport in superfluid [26,27] in addition to superconducting charge transport.

The multifaceted aspect of spin-triplet superconductivity also gives rise to additional complexity in fluctuations. An especially telling case would be the spin-triplet superconductor in two dimensions (2D) with easy-plane anisotropy, which, as we shall show, has the likespin pairing when the spin quantization axis is perpendicular. Such two component condensates in 2D would allow multiple types of Berezinskii-Kosterlitz-Thouless (BKT) phase transitions $[28,29]$ involving both the charge and the spin degrees of freedom. While the effects of vortex fluctuations on charge transport in superconductors have now been understood well [30], the effects of the critical fluctuations on charge and spin transport in spin-triplet superconductors have not been studied yet. In particular, since spin-triplet superconductors have been proposed as efficient spin-transport medium in superconducting spintronics, it would be crucial to investigate the robustness of superfluid spin transport in 2D spin-triplet superconductors for realizing superconductor-based spintronics with minimal dissipation [31]. Previously, we have shown that quasi-long range ordering is sufficient for spin superfluid transport in the 2D XY magnets [32]. Whether the same robustness exists for spin transport in spin-triplet superconductors has remained an open question.

In this Letter, we study the effects of critical fluctuations associated with three distinct types of vortices-conventional vortex, $\mathbf{d}$-vector meron, and fractional vortex-on charge and spin transport in equal-spin-pairing spin-triplet superconductors. Specifically, we show that superfluid spin transport of spin-triplet superconductors at finite temperatures fundamentally differs from that of $\mathrm{XY}$ magnets due to the existence of fractional vortices, which are topological defects intertwining charge and spin currents. More specifically, we show that the fractional vortex sets an upper bound to spin current, and this upper bound decreases algebraically with distance. Our results indicate the possibility of transport detection of fractional vortices in spin-triplet superconductors. Also, the identified vulnerability of superfluid 
spin transport to topological defects calls for further investigations of fluctuation effects on promised superconductor-based spintronic devices.

\section{General considerations}

Due to its $U(1) \times U(1)$ order parameter, three types of vortices, with therefore three types of BKT transitions in 2D, should be considered for the easy-plane spin-triplet superconductor. This form of order parameter arises because the Cooper pair condensation in the spin-triplet superconductor necessarily involves the ordering of the Cooper pair spin state, as shown by the multi-component pairing gap [1-4]

$$
i(\mathbf{d} \cdot \boldsymbol{\sigma}) \sigma_{y}=\left[\begin{array}{cc}
-d_{x}+i d_{y} & d_{z} \\
d_{z} & d_{x}+i d_{y}
\end{array}\right] \equiv\left[\begin{array}{cc}
\Delta_{\uparrow \uparrow} & \Delta_{\uparrow \downarrow} \\
\Delta_{\downarrow \uparrow} & \Delta_{\downarrow \downarrow}
\end{array}\right],
$$

in the absence of the condensate spin-polarization, the $\mathbf{d}$-vector is real up to an overall phase. For the case where the direction of the $\mathbf{d}$-vector lies in the $x y$-plane, i.e. $\hat{\mathbf{d}}=(\cos \alpha, \sin \alpha, 0)$, Eq. (1) shows clearly that only the diagonal elements are non-zero with $\Delta_{\sigma \sigma}=|\Delta| e^{i \pi \frac{1+\sigma}{2}} e^{i(\theta-\sigma \alpha)}$, where $\theta$ is the overall phase. Hence the Cooper pair charge and spin current would be proportional to $\nabla \theta-\frac{2 e}{\hbar c} \mathbf{A}$ and $\nabla \alpha$, respectively [25, 33]. As this $\mathrm{U}(1) \times \mathrm{U}(1)$ order parameter remains single-valued for vortices with $m^{\mathrm{c}} \mp m^{\mathrm{sp}} \in \mathbb{Z}$, where $m^{\mathrm{c}}\left(m^{\mathrm{sp}}\right)$ is the number of $2 \pi$ windings of charge (spin) phase $\theta(\alpha)$, the basic topological defects supported by the easy-plane spin-triplet superconductor include not only the conventional full-quantum vortex (fqv) with $\left(m^{\mathrm{c}}, m^{\mathrm{sp}}\right)=( \pm 1,0)$ and the $d$-vector meron $(\mathrm{dm})$ with $\left(m^{\mathrm{c}}, m^{\mathrm{sp}}\right)=(0, \pm 1)$, but, as illustrated in Fig. 1, also the half-quantum vortex (hqv) with $\left|m^{\mathrm{c}}\right|=\left|m^{\mathrm{sp}}\right|=1 / 2$ [34-38]. The existence of half-quantum vortices in spin-triplet superconductors is due to the fact that the order-parameter matrix [Eq. (1)] remains single-valued by simultaneously rotating the $\mathbf{d}$ vector by $\pi$ (i.e., $\alpha \rightarrow \alpha \pm \pi$ ) while winding the overall phase by $\pi$ (i.e., $\theta \rightarrow \theta \pm \pi$ ). It has been shown that fractional vortices can exist for this type of superconducting order parameter even when its physical mechanism is entirely different [39-44]. Given that the BKT transition arises from the (de)confinement of vortices below (above) the transition temperature, the existence of three types of vortices implies the possibility of three distinct types of the BKT transitions in the 2D easy-plane spin-triplet superconductor (see Fig. 2).

These BKT transitions can be conveniently studied by treating vortices as particles [32,4549]. For the $2 \mathrm{D}$ easy-plane spin-triplet superconductor, its $\mathrm{U}(1) \times \mathrm{U}(1)$ order parameter allows us to study the energetics of quenched vortices from the Coulomb gas action $[35,50]^{1}$

$$
\begin{aligned}
S_{\text {eff }}= & 2 \pi \hbar \sum_{i} \int^{\mathbf{r}_{i}} d \mathbf{r}^{\prime} \cdot\left[\frac{m_{i}^{\mathrm{c}}}{2 e} \mathbf{J}_{\mathrm{c}}^{\mathrm{ext}}\left(\mathbf{r}^{\prime}\right)-\frac{m_{i}^{\mathrm{sp}}}{\hbar} \mathbf{J}_{\mathrm{sp}}^{z, \text { ext }}\left(\mathbf{r}^{\prime}\right)\right] \times \hat{\mathbf{z}} \\
& -2 \pi \sum_{i \neq j}\left(m_{i}^{\mathrm{c}} m_{j}^{\mathrm{c}} K_{\mathrm{c}}+m_{i}^{\mathrm{sp}} m_{j}^{\mathrm{sp}} K_{\mathrm{sp}}\right) \log \frac{\left|\mathbf{r}_{i}-\mathbf{r}_{j}\right|}{\xi},
\end{aligned}
$$

where the first term arises from the transverse Magnus force that external current applies on vortices with $\mathbf{J}_{\mathrm{c}}^{\text {ext }}\left(\mathbf{J}_{\mathrm{sp}}^{z \text {,ext }}\right.$ ) being the externally applied charge (spin) current, while the second term is the vortex-vortex interaction energy with $\xi$ being the vortex core radius and $K_{\mathrm{c}}\left(K_{\mathrm{sp}}\right)$ the phase stiffness for charge (spin). Here, the charge and the spin winding number, $m_{i}^{\mathrm{c}}$ and $m_{i}^{\mathrm{sp}}$, can be either both integers or both half-integers depending on the nature of vortices.

\footnotetext{
${ }^{1}$ In chiral superconductor, additional topological terms may be present, giving rise to the non-Abelian hqv braid statistics [51] plus a universal Abelian vortex exchange phase factor [52], neither of which, however, contribute to energetics of the vortex pair unbinding.
} 


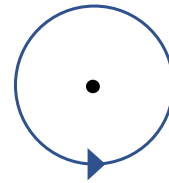

$\Delta \theta=2 \pi$

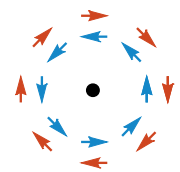

$\Delta \varphi_{\uparrow}=2 \pi$

$\Delta \varphi_{\downarrow}=2 \pi$

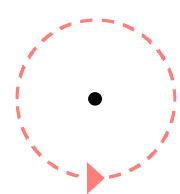

$\Delta \alpha=2 \pi$

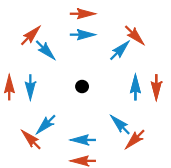

$\Delta \varphi_{\uparrow}=-2 \pi$

$\Delta \varphi_{\downarrow}=2 \pi$
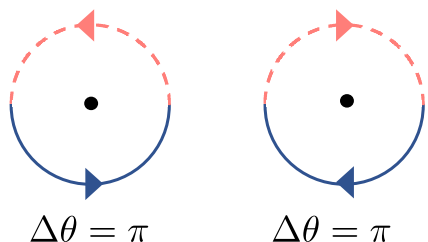

$\Delta \theta=\pi$

$\Delta \alpha=\pi$

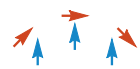

$\uparrow \uparrow \bullet \uparrow \uparrow$

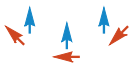

$\Delta \varphi_{\uparrow}=0$

$\Delta \varphi_{\downarrow}=2 \pi$

(b)
$\Delta \alpha=-\pi$

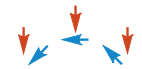

$\downarrow \downarrow \bullet \uparrow \downarrow$

$r \rightarrow d$

$\Delta \varphi_{\uparrow}=2 \pi$

$\Delta \varphi_{\downarrow}=0$ (c)

(a)

Figure 1: Phase windings of (a) a full-quantum vortex (fqv), (b) a $d$-vector meron (dm), and (c) half-quantum vortices (hqv), where $\theta$ and $\alpha$ represent phases associated with charge and spin order, respectively, and $\varphi_{\sigma}$ represent the phase of the spin- $\sigma$ Cooper pair $\Delta_{\sigma \sigma}=\left|\Delta_{\sigma \sigma}\right| e^{i \varphi_{\alpha}}$. A fqv, a dm, and a fqv have U(1) winding numbers $\left(m^{\mathrm{c}}, m^{\mathrm{sp}}\right)=( \pm 1,0),(0, \pm 1)$, and $( \pm 1 / 2, \pm 1 / 2)$, respectively, where $m^{\mathrm{c}}$ and $m^{\mathrm{sp}}$ are the winding numbers associated with $\theta$ and $\alpha$.

Since the BKT transition temperature is determined by the interaction between vortices, from the second term of Eq. (2), the BKT temperature for each vortex type can be determined by adopting the known results for superfluids and superconductors [30,41, 50, 53, 54]:

$$
T_{\mathrm{BKT}}\left(\left|m^{\mathrm{c}}\right|,\left|m^{\mathrm{sp}}\right|\right)=\frac{\pi}{2 k_{B}}\left[\left(m^{\mathrm{c}}\right)^{2} K_{\mathrm{c}}+\left(m^{\mathrm{sp}}\right)^{2} K_{\mathrm{sp}}\right],
$$

which yields

$$
T_{\mathrm{BKT}}^{\mathrm{fqv}}=\frac{\pi K_{c}}{2 k_{B}}, \quad T_{\mathrm{BKT}}^{\mathrm{dm}}=\frac{\pi K_{s}}{2 k_{B}}, \quad T_{\mathrm{BKT}}^{\mathrm{hqv}}=\frac{\pi\left(K_{c}+K_{s}\right)}{8 k_{B}},
$$

for the BKT transitions driven by proliferations of fqv with $\left(m^{\mathrm{c}}, m^{\mathrm{sp}}\right)=( \pm 1,0)$, dm with $\left(m^{\mathrm{c}}, m^{\mathrm{sp}}\right)=(0, \pm 1)$, and hqv with $\left(m^{\mathrm{c}}, m^{\mathrm{sp}}\right)=( \pm 1 / 2, \pm 1 / 2)$, respectively. ${ }^{2}$ Upon increasing the temperature, among the three possible BKT transitions, the one with the lowest critical temperature would disorder the spin-triplet superconductivity, and it is determined by, as shown in Fig. 2, the stiffness ratio $K_{\mathrm{sp}} / K_{\mathrm{c}}: K_{\mathrm{sp}} / K_{\mathrm{c}}>3, K_{\mathrm{sp}} / K_{\mathrm{c}}<1 / 3$, and $1 / 3<K_{\mathrm{sp}} / K_{\mathrm{c}}<3$ correspond to the fqv, the $\mathrm{dm}$, and the hqv deconfinement, respectively ${ }^{3}$. It is possible to derive from Eq. (2), together with phenomenological vortex mobility that we shall introduce below, the DC transport change at the BKT transitions from the presence (absence) of the free vorticity density $n_{\mathrm{c} / \mathrm{sp}}^{f}$ above (below) the transition.

\footnotetext{
${ }^{2}$ Vortices with finite charge-phase winding number can harbor a finite spin polarization, as discussed in Ref. [55] in the context of vortex-flux flow spin Hall effect in superconductors with spin-splitting field. However, the BKT transitions that are of interest to us in our work are governed by long-range interactions between vortices, rather than properties of individual vortices. For this reason, we expect that while the local coupling between the charge vorticity and the spin density can affect properties of individual vortices, it would not change the physics of the BKT transitions discussed in our work qualitatively.

${ }^{3}$ Possible complications from thermal fluctuation of more than two vortex types are ignored in this work.
} 


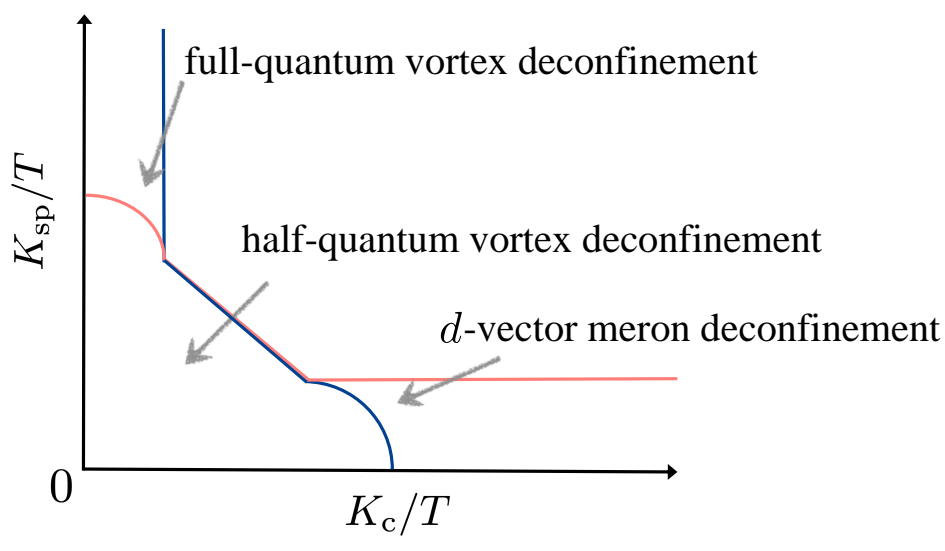

Figure 2: Three distinct Bereziskii-Kosterlitz-Thouless transitions driven by deconfinements of three different types vortices in the $\left(K_{\mathrm{sp}} / T, K_{\mathrm{c}} / T\right)$ plane, where the arrow directions correspond to increasing temperature and the blue (red) curve indicates phase transition in the charge (spin) sector. Deconfinements of full-quantum vortex and $d$-vector meron leads to the exponentially decaying phase coherence of charge order and spin order, respectively, whereas deconfinement of half-quantum vortex the exponentially decaying phase coherence of both orders.

Specifically, above the transition temperature, where the free vortex density is finite, we assume for simplicity that the vortex mobility to be purely longitudinal, i.e. the vortex current density is obtained by multiplying the vortex mobility $\mu$ to the transverse Magnus force that can be derived from the first term of Eq. (2):

$$
\mathbf{j}\left(m^{\mathrm{c}}, m^{\mathrm{sp}}\right)=2 \pi \hbar \mu n^{f}\left[\frac{\operatorname{sgn}\left(m^{\mathrm{c}}\right)}{2 e} \mathbf{J}_{\mathrm{c}}^{\mathrm{ext}}-\frac{\operatorname{sgn}\left(m^{\mathrm{sp}}\right)}{\hbar} \mathbf{J}_{\mathrm{sp}}^{z, \mathrm{ext}}\right] \times \hat{\mathbf{z}},
$$

where $n^{f}$ is the free vortex density; we take $\operatorname{sgn}(0)=0$. The contribution of this vortex current to the charge/spin vorticity current is $\mathbf{j}_{\mathrm{c} / \mathrm{sp}}=\mathrm{m}^{\mathrm{c} / \mathrm{sp}} \mathbf{j}\left(\mathrm{m}^{\mathrm{c}}, \mathrm{m}^{\mathrm{sp}}\right)$. Note that the linear relationship between the charge/spin current and the charge/spin vorticity current holds above the transition temperature.

Below the BKT transition, where free vortices are absent, the energy barrier against the vortex pair unbinding is finite in the presence of an external current density, for the vortex pair energy, rather than increasing monotonically with distance, reaches its maximum when the inverse distance pair attraction is at equilibrium with the Magnus force applied by the external current, as can be derived from Eq. (2). The energy barrier per vortex would be half of this maximum, which, for weak external currents, is

$$
\Delta E \approx 2 k_{B} T_{\mathrm{BKT}}\left(\left|m^{\mathrm{c}}\right|,\left|m^{\mathrm{sp}}\right|\right) \log \frac{2 k_{B} T_{\mathrm{BKT}}\left(\left|m^{\mathrm{c}}\right|,\left|m^{\mathrm{sp}}\right|\right)}{\pi \hbar \xi\left|\frac{m^{\mathrm{c}}}{2 e} J_{\mathrm{c}}^{\mathrm{ext}}-\frac{m^{\mathrm{sp}}}{\hbar} J_{\mathrm{sp}}^{z \text { ext }}\right|} .
$$

This implies thermal dissociation probability $\propto \exp \left(-\Delta E / k_{B} T\right)$ for bound vortex pairs. Therefore, the current $\mathbf{j}\left(\mathrm{m}^{\mathrm{c}}, \mathrm{m}^{\mathrm{sp}}\right)$ is linear in applied current above the transition but nonlinear with the exponent of $1+2 T_{\mathrm{BKT}} / T$ below the transition $[30,32,53]$, and the transport signature of the BKT transition arises from this change of vortex current.

Yet, due to the finite spin lifetime, the effect of a vortex current on transport properties differs qualitatively between the charge and the spin sector. First, for the charge sector, charge conservation dictates that incoming and outgoing current should be equal. The electric field $\mathbf{E}$ induced by the charge vortex current density $\mathbf{j}_{c}$ can be derived from the second Josephson 


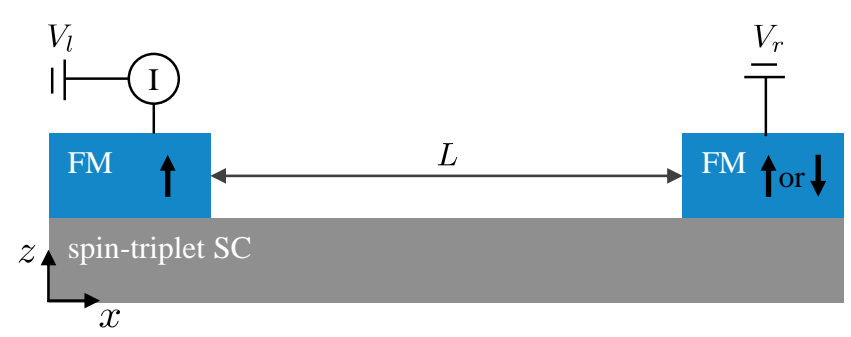

Figure 3: Illustration of an experimental setup for transport experiment, where a spin-triplet superconductor (SC) is used as a charge- and spin-transport medium and two ferromagnetic metals (FMs) serve as magnetic leads with distance $L$.

relation [56],

$$
\mathbf{E}=2 \pi \frac{\hbar}{2 e} \hat{\mathbf{z}} \times \mathbf{j}_{\mathrm{c}}=\frac{\pi \hbar}{e} \hat{\mathbf{z}} \times \sum_{m^{\mathrm{c}}, m^{\mathrm{sp}}} m^{\mathrm{c}} \mathbf{j}\left(m^{\mathrm{c}}, m^{\mathrm{sp}}\right),
$$

indicating that the qualitative change of current-voltage relation across the BKT transition represents the corresponding change of the charge vorticity current. Second, for the spin sector, the spin analogue of the second Josephson relation Eq. (5) $[32,49,57,58]$ gives us $\nabla s_{z}=2 \pi\left(K_{\mathrm{sp}} / v_{\mathrm{sp}}^{2}\right) \hat{\mathbf{z}} \times \mathbf{j}_{\mathrm{sp}}$, where $s_{z}$ is the spin density of Cooper pairs and $v_{\mathrm{sp}}$ is the spin-mode velocity. However, while charge conservation ensures uniform DC charge current, the same does not hold for spin due to the finite lifetime of spin. Indeed, $\boldsymbol{\nabla} \cdot \mathbf{J}_{\mathrm{sp}}^{z}+s_{z} / \tau=0$ for DC transport $[32,33]$ with $\tau$ spin lifetime leads to

$$
\boldsymbol{\nabla}\left(\boldsymbol{\nabla} \cdot \mathbf{J}_{\mathrm{sp}}\right)=\frac{1}{\tau} \nabla s_{z}=2 \pi \frac{K_{\mathrm{sp}}}{\tau \nu_{\mathrm{sp}}^{2}} \mathbf{j}_{\mathrm{sp}} \times \hat{\mathbf{z}}
$$

This implies that the change in the spin vorticity current $\mathbf{j}_{\mathrm{sp}}$ across the BKT transition can be detected by the change in the spatial profile of the spin current $\mathbf{J}_{\mathrm{sp}}$, which, as we shall show below, manifests through the dependence of magnetoresistance on the distance between the source and drain of spin current.

\section{Transport signature of BKT transitions}

\subsection{Transport setup}

The distinct signature of each BKT transition can be detected from the DC current-voltage relation for the setup of Fig. 3, which has been used in Ref. [25] to study zero-temperature magnetoresistance of spin-triplet superconductors. The proposed setup is referred to as a spinvalve structure, which allows for electrical measurement of spin transport and thus has been widely used in spintronics to study spin-dependent transport properties of magnetic metals. In this work, we are interested in the changes of charge and spin transport across three types of BKT transitions, and these can be detected through spin-dependent current-voltage relation in the proposed setup as will be detailed below. The proposed setup is referred to as a spinvalve structure, which allows for electrical measurement of spin transport and thus has been widely used in spintronics to study spin-dependent transport properties of magnetic metals. In this work, we are interested in the changes of charge and spin transport across three types of BKT transitions, and these can be detected through spin-dependent current-voltage relation in the proposed setup as will be detailed below. It consists of two leads made of ferromagnetic metal (e.g. $\left.\mathrm{SrRuO}_{3}\right)$ attached to the spin-triplet superconductor, with the lead magnetization 
perpendicular to the superconductor $\mathbf{d}$-vector. One possible material candidate is the recently fabricated $\mathrm{SrRuO}_{3} \mid \mathrm{Sr}_{2} \mathrm{RuO}_{4}$ heterostructure [59]. The logical starting point for deriving the bulk current-voltage relation that will be measured in the setup of Fig. 3 is the boundary condition for the current of spin- $\sigma$ Cooper pairs,

$$
I_{l, r}^{\sigma}= \pm g_{l, r}^{\sigma \sigma}\left(V_{l, r}-\frac{\hbar}{2 e} \partial_{t} \varphi_{\sigma}^{l, r}\right)
$$

( $g_{l, r}^{\sigma \sigma}$ is the spin- $\sigma$ conductance for the left/right lead, $V_{l, r}$ the voltage on the normal side of the left/right lead, and $\varphi_{\sigma} \equiv \arg \Delta_{\sigma \sigma}$ ), where, the second Josephson relation is modified by the voltage drop at the contact. Adding and subtracting the two spin components in Eq. (7) gives us the spin current boundary condition

$$
I_{l, r}^{\mathrm{sp}}=\frac{\hbar}{2 e} p_{l, r} I \pm\left(1-p_{l, r}^{2}\right) \frac{\hbar^{2}}{4 e^{2}} \partial_{t} \alpha_{l, r}=\frac{\hbar}{2 e} p_{l, r} I \pm\left(1-p_{l, r}^{2}\right) \tilde{g}_{l, r} \frac{d}{d x} I_{l, r}^{\mathrm{sp}},
$$

where $\tilde{g}_{l, r} \equiv \frac{\hbar^{2}}{4 e^{2}} \frac{K_{\mathrm{sp}}}{v_{\mathrm{sp}}^{2}} \frac{\tau}{w} g_{l, r}$ ( $w$ is the lead width and $g_{l, r} \equiv \sum_{\sigma} g_{l, r}^{\sigma \sigma}$ ), and we have used $\partial_{t} \alpha_{l, r}=-\frac{K_{\mathrm{sp}}}{v_{\mathrm{sp}}^{2}} s_{z}^{l, r}=\frac{K_{\mathrm{sp}}}{v_{\mathrm{sp}}^{2}} \frac{\tau}{w} \frac{d}{d x} I_{l, r}^{\mathrm{sp}}$, derived from the finite spin lifetime and $\left[s_{z}(\mathbf{r}), \alpha\left(\mathbf{r}^{\prime}\right)\right]=i \delta\left(\mathbf{r}-\mathbf{r}^{\prime}\right)$; note that, as shown in Fig. 3, the current is constrained to flow along the $x$-direction, with $x=\mp L / 2$ for the left / right lead. Again by adding and subtracting Eq. (7), we obtain the bulk current-voltage relation,

$$
\Delta V \equiv\left(V_{l}-V_{r}\right)-\frac{g_{l}+g_{r}}{g_{l} g_{r}} I=\frac{\hbar}{2 e}\left(\partial_{t} \theta_{l}-\partial_{t} \theta_{r}\right)-\frac{\hbar}{2 e} \frac{K_{\mathrm{sp}}}{v_{\mathrm{sp}}^{2}} \frac{\tau}{w}\left(p_{l} \frac{d}{d x} I_{l}^{\mathrm{sp}}-p_{r} \frac{d}{d x} I_{r}^{\mathrm{sp}}\right)
$$

where $p_{l, r} \equiv \sum_{\sigma} \sigma g_{l, r}^{\sigma \sigma} / g_{l, r}$ is the contact conductance spin polarization. The novelty in our BKT transport arises from the second term of Eq. (9) that gives the magnetoresistance through $p_{l, r}$, which, as we shall show, allows for electrical detection of the spin vorticity deconfinement.

\subsection{Full-quantum vortex deconfinement}

While the fqv deconfinement, as it consists of the charge vorticity deconfinement alone, is of the same type as the BKT transition of conventional superconductors with respect to charge transport [30,53,60-62], spin transport reveals the fqv deconfined phase to be unconventional. In the case of the fqv deconfinement, the change in the first term of $\Delta V$ in Eq. (9), $\left(\partial_{t} \theta_{l}-\partial_{t} \theta_{r}\right) / 2 \pi=\int_{-L / 2}^{+L / 2} d x j_{c}$, through the transition is indeed exactly that of the BKT transition in conventional superconductor. However, the voltage-current relation across the fqv deconfinement is modified by the second term in Eq. (9) involving the spin current $I^{\mathrm{sp}}$, which cannot be absent in the Fig. 3 setup.

In the considered setup, due to the absence of spin vorticity both above and below the transition, the spin torque always vanishes in the bulk, leading to a uniform $s_{z}$, and hence a spin current linear in the distance $x$ from the leads $[25,33]$. The linear profile of the spin current, together with Eq. (8) gives us

$$
\frac{\frac{\hbar}{2 e}\left(p_{l}-p_{r}\right) I}{d I^{\mathrm{sp}} / d x}=-L-\left[\left(1-p_{l}^{2}\right) \tilde{g}_{l}+\left(1-p_{r}^{2}\right) \tilde{g}_{r}\right],
$$

and inserting this into Eq. (9) gives us the following current-voltage relation in the large- $L$ limit,

$$
\Delta V=\frac{\hbar}{2 e} \frac{K_{\mathrm{sp}}}{v_{\mathrm{sp}}^{2}} \frac{\tau}{w} \frac{\left(p_{l}-p_{r}\right)^{2}}{L} I+\left\{\begin{array}{cl}
\frac{L}{w} \rho_{0}^{\mathrm{fqv}} I\left|\frac{I}{I_{0}^{\mathrm{fqv}}}\right|^{2 \mathrm{~T}_{\mathrm{BKT}}^{\mathrm{fqv}} / T} & \left(T<T_{\mathrm{BKT}}^{\mathrm{fqv}}\right), \\
\frac{L}{w}\left(\frac{\pi \hbar}{e}\right)^{2} \mu_{\mathrm{fqv}} n_{f} I & \left(T>T_{\mathrm{BKT}}^{\mathrm{fqv}}\right),
\end{array}\right.
$$




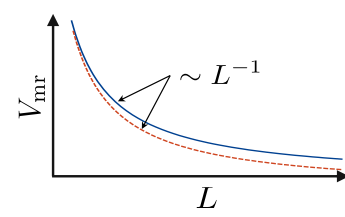

(a)

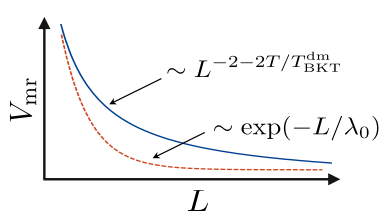

(b)

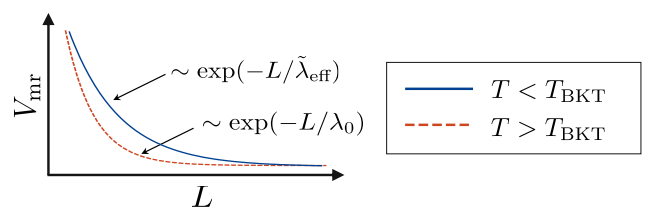

(c)

Figure 4: Schematic illustrations of the length dependence of the voltage magnetoresistance $V_{\mathrm{mr}} \equiv \Delta V\left(p_{l}=-p_{r}=p\right)-\Delta V\left(p_{l}=p_{r}=p\right)$ below and above the BKT transition temperatures driven by proliferations of (a) full-quantum vortices [Eq. (10)], (b) $d$-vector merons [Eqs. $(11,12)]$, and (c) half-quantum vortices [Eqs. $(14,15)]$. See the main text for detailed discussions.

where $\rho_{0}^{\text {fqv }}$ and $I_{0}^{\text {fqv }}$ are phenomenological constants in the units of $2 \mathrm{D}$ resistivity and current, respectively ${ }^{4}$; this is our first main result. Below or above $T_{\mathrm{BKT}}^{\mathrm{fqv}}$, the current-voltage relation in absence of the spin polarization is exactly that of the conventional BKT transition. The difference lies in magnetoresistance $V_{\mathrm{mr}} \equiv \Delta V\left(p_{l}=-p_{r}=p\right)-\Delta V\left(p_{l}=p_{r}=p\right)$, which remains unchanged across the BKT transition and reproduces the result of Ref. [25]: $V_{\mathrm{mr}} \propto I / L$. This implies that, as indicated in Fig. 4, we have an unconventional phase above the transition that, while not superconducting, retains some of spin quasi-ordering from the spin-triplet superconductivity $[50,63]$. This is because, when only the overall phase is disordered, there still remains spin nematicity, defined from the order parameter of Eq. (1) as quasi-long range ordered $\Delta_{\uparrow \uparrow} \Delta_{\downarrow \downarrow}^{*} \propto\left(\hat{d}_{x}^{2}-\hat{d}_{y}^{2}\right)-i 2 \hat{d}_{x} \hat{d}_{y}$.

\section{$3.3 d$-vector meron deconfinement}

Conversely, the deconfinement of spin vortices, i.e. dm, directly disturbs spin transport (as merons interrupt spin transport in 2D XY magnets [32]), giving rise to the change in the spin transport equation, i.e.

$$
\frac{I_{0}^{\mathrm{sp}}}{I^{\mathrm{sp}}} \frac{d^{2}}{d x^{2}} \frac{I^{\mathrm{sp}}}{I_{0}^{\mathrm{sp}}}=\left\{\begin{array}{cc}
\lambda^{-2}\left|\frac{I^{\mathrm{sp}}}{I_{0}^{\mathrm{sp}}}\right|^{2 T_{\mathrm{BKT}}^{\mathrm{dm}} / T} & \left(T<T_{\mathrm{BKT}}^{\mathrm{dm}}\right), \\
\lambda_{0}^{-2} & \left(T>T_{\mathrm{BKT}}^{\mathrm{dm}}\right),
\end{array}\right.
$$

where $I_{0}^{\mathrm{sp}}$ and $\lambda$ are phenomenological constants in the units of spin current and length, respectively, and $\lambda_{0}=\sqrt{\frac{v_{\mathrm{sp}}^{2} \tau}{\mu_{\mathrm{dm}} n_{f}}}$ is the spin diffusion length derived from Eqs. (3) and (6) [32]. Meanwhile the absence of the charge vorticity current means $\left(\partial_{t} \theta_{l}-\partial_{t} \theta_{r}\right) / 2 \pi=\int_{-L / 2}^{+L / 2} d x j_{c}=0$ holds across the transition. Hence, from Eq. (9), we can see that this transition would manifest in the current-voltage relation only through the change in magnetoresistance. Although Eq. (10) below the transition is nonlinear, our analysis is facilitated by its being readily reducible to a first-order differential equation for the symmetric (antisymmetric) lead configuration, $p_{l}= \pm p_{r}$ with $g_{l}=g_{r}=g$.

By solving the bulk equations of motion in conjunction with charge and spin boundary conditions in the large- $L$ limit, and keeping only the leading-order functional dependence on

\footnotetext{
${ }^{4}$ Vortices with the opposite winding may not have the same mobility in the chiral superconductor; in that case $\mu_{\mathrm{fqv}}$ should be interpreted as the average mobility.
} 
$I$ and $L$, we obtain the following magnetoresistance:

$$
V_{\mathrm{mr}} \propto\left(\frac{L}{\lambda}\right)^{-2-\frac{2 T}{T_{\mathrm{BKT}}^{\mathrm{dm}}}}\left|\frac{\hbar I}{2 e I_{0}^{\mathrm{sp}}}\right|^{-1-\frac{T_{\mathrm{BKT}}^{\mathrm{dm}}}{T}}
$$

for $T \lesssim T_{\mathrm{BKT}}^{\mathrm{dm}}$ in the $L \gg \lambda\left|e I_{0}^{\mathrm{sp}} / p \hbar I\right|^{\frac{T_{\mathrm{BKT}}^{\mathrm{dm}}}{T}}$ limit and

$$
V_{\mathrm{mr}} \propto I e^{-L / \lambda_{0}}
$$

for $T>T_{\mathrm{BKT}}^{\mathrm{dm}}$, which constitute our second main result. It indicates that for $T<T_{\mathrm{BKT}}^{\mathrm{dm}}$ the magnetoresistance decreases algebraically with $L$ as $V_{\mathrm{mr}} \sim L^{-2-2 T / T_{\mathrm{BKT}}^{\mathrm{dm}}}$, but vanishes exponentially with $L$ for $T>T_{\mathrm{BKT}}^{\mathrm{dm}}$ with the decay length given by the spin diffusion length $\lambda_{0}$ defined above. This is qualitatively equivalent to the change of spin transport across the magnetic BKT transition in easy-plane magnets [32]. The complete expressions, as shown in Appendix $\mathrm{B}$, also gives us the vanishing of all dissipation in absence of any spin-polarization, i.e. $\Delta V\left(p_{l}=p_{r}=0\right)=0$, on both sides of the transition. This can be taken as the evidence for the persistence of superconductivity, i.e. absence of dissipation from the charge degree of freedom, above the dm BKT transition where spin ordering is destroyed, as indicated in Fig. 4. This indicates a charge-4e superconductivity involving pairing of two Cooper pairs, i.e. $\Delta_{\uparrow \uparrow} \Delta_{\downarrow \downarrow} \propto e^{i 2 \theta}[15,41]$.

\subsection{Half-quantum vortex deconfinement}

As an hqv possesses vorticity in both charge and spin, separation of the charge and the spin transport is not guaranteed in presence of the hqv current. This can be revealed only through spin-polarized current bias; otherwise transport effects of hqv's will not differ qualitatively from those of fqv's, as one can infer from Eq. (2). To treat this, it needs to be noted that two types of hqv's, each with $m^{\mathrm{c}}-\sigma m^{\mathrm{sp}}= \pm 1$ and therefore, as shown in Fig. 1 (c), a nonzero vorticity only for $\Delta_{\sigma \sigma}$, both have deconfinement onset at $T=T_{\mathrm{BKT}}^{\mathrm{hqv}}$ in absence of bulk spinpolarization, but their contributions to the spin vorticity current have opposite signs. Hence, for $T<T_{\mathrm{BKT}}^{\mathrm{hqv}}$,

$$
j_{\mathrm{sp}}=\frac{\hbar v_{\mathrm{sp}}^{2} \tau}{4 \pi K_{\mathrm{sp}} w \tilde{\lambda}^{2}} \sum_{\sigma} \sigma \frac{I+\sigma \frac{2 e}{\hbar} I^{\mathrm{sp}}}{2 e}\left|\frac{I+\sigma \frac{2 e}{\hbar} I^{\mathrm{sp}}}{I_{0}^{\mathrm{hqv}}}\right|^{\frac{2 T_{\mathrm{BKT}}^{\mathrm{hq}}}{T}},
$$

where $I_{0}^{\mathrm{hqv}}$ and $\tilde{\lambda}$ are phenomenological constants in the unit of current and length. Combined with Eq. (6), this leads to the spin current differential equation

$$
\frac{d^{2}}{d x^{2}} \frac{I^{\mathrm{sp}}}{\frac{\hbar}{2 e} I_{0}^{\mathrm{hqv}}} \approx \frac{1+\frac{2 T_{\mathrm{BKT}}^{\mathrm{hqv}}}{T}}{\tilde{\lambda}^{2}}\left|\frac{I}{I_{0}^{\mathrm{hqv}}}\right|^{\frac{2 T_{\mathrm{BKT}}^{\mathrm{hgv}}}{T}} \frac{I^{\mathrm{sp}}}{\frac{\hbar}{2 e} I_{0}^{\mathrm{hqv}}},
$$

for most of the bulk in the large- $L$ limit, as the finite spin lifetime leads to $I^{\mathrm{sp}} \ll \frac{\hbar}{2 e} I$. For the same reason, the charge vorticity current in this limit can be taken to be nearly independent of the spin current, $j_{c} \propto|I|^{1+2 T_{\mathrm{BKT}}^{\mathrm{hqV}} / T}$. This indicates that, while the bound hqv's behave like the bound fqv's in the charge sector, they induce, in contrast to the bound dm's, the exponential decay of the spin current when the charge current has a finite magnitude, with the spin decay length of

$$
\tilde{\lambda}_{\mathrm{eff}} \equiv \frac{\tilde{\lambda}}{\sqrt{1+2 T_{\mathrm{BKT}}^{\mathrm{hqv}} / T}}\left|\frac{I_{0}^{\mathrm{hqv}}}{I}\right|^{\substack{T_{\mathrm{BRT}}^{\mathrm{hqv}} \\ T}} .
$$


For this case, we have the magnetoresistance voltage:

$$
V_{\mathrm{mr}} \sim I e^{-L / \tilde{\lambda}_{\mathrm{eff}}} \quad \text { for } T<T_{\mathrm{BKT}}^{\mathrm{hqv}},
$$

which decays exponentially as a function of the lead spacing $L$ with the length scale given by $\tilde{\lambda}_{\text {eff }}$. Since the decaying length $\tilde{\lambda}_{\text {eff }}$ diverges as $I \rightarrow 0$, the long-range spin transport do survive

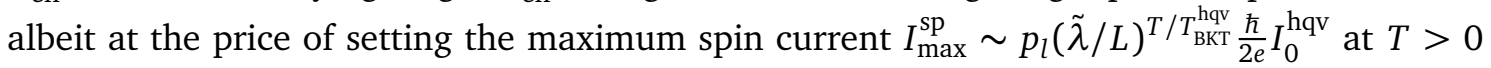
for the given lead spacing $L$.

For $T>T_{\mathrm{BKT}}^{\mathrm{hqv}}$, by contrast, it is possible to separate the charge and the spin sector, i.e. the charge (spin) vorticity current is proportional to the charge (spin) current and independent of the spin (charge) current as the densities of all hqv types are equal and current independent to the leading order. Therefore, above the transition, we have

$$
V_{\mathrm{mr}} \propto I e^{-L / \tilde{\lambda}_{0}} \quad \text { for } T>T_{\mathrm{BKT}}^{\mathrm{hqv}},
$$

where $\tilde{\lambda}_{0}^{2} \equiv \frac{v_{\mathrm{sp}}^{2} \tau}{\mu_{\mathrm{hqv}} n_{f}}$. The magnetoresistance voltage decays exponentially as a function of $L$ akin to the low-temperature case, but the decaying length $\tilde{\lambda}_{0}$ is finite in the limit of $I \rightarrow 0$. Eqs. (15), (16) are our third main results. For the full expressions of $V_{\mathrm{mr}}$ for the hqv case, see Appendix C.

\section{Conclusion}

We have explained how the hqv-driven BKT transition displays transport characteristics absent in the fqv-driven or dm-driven BKT transitions. Experimental detection for fixed $L$ can be made by examining how $V_{\mathrm{mr}} / I$ ratio depends on $I$. For $T>T_{\mathrm{BKT}}^{\mathrm{hqv}}, L>\tilde{\lambda}_{0}$ ensures exponentially vanishing $V_{\mathrm{mr}} / I$ independent of $I$. For $T<T_{\mathrm{BKT}}^{\mathrm{hqv}}, V_{\mathrm{mr}} / I$ will not be exponentially small for $I$ smaller than the critical value that scales as $L^{-T / T_{\mathrm{BKT}}^{\mathrm{hqv}}}$.

We expect our results to be applicable to many of superconductors where $U(1) \times U(1)$ order parameters arise from various mechanisms [39-44]. All these superconductors can be regarded as having a two component condensates, and our hqv transport results of Eqs. (15) and (16) should hold if in-plane anisotropy is very weak or arising from the hexagonal crystal field [64] (a frequent feature in van der Waals materials) and any imbalance between the two components has a finite relaxation time.

\section{Acknowledgements}

We would like to thank Yaroslav Tserkovnyak, Steve Kivelson, Eduardo Fradkin, and S. Raghu and for sharing their insights into the BKT physics, and Tae Won Noh for motivating this work. S.B.C. was supported by the National Research Foundation of Korea (NRF) grants funded by the Korea government (MSIT) (2020R1A2C1007554) and the Ministry of Education (2018R1A6A1A06024977). S.K.K. was supported by Brain Pool Plus Program through the National Research Foundation of Korea (NRF) funded by the Ministry of Science and ICT (2020H1D3A2A03099291), by the National Research Foundation of Korea (NRF) grant funded by the Korea government (MSIT) (2021R1C1C1006273), and by the National Research Foundation of Korea funded by the Korea Government via the SRC Center for Quantum Coherence in Condensed Matter (2016R1A5A1008184). S. K. K. was also supported by Basic Science Research Program through the KAIST Basic Science 4.0 Priority Research Center funded by the Ministry of Science and ICT. 


\section{A The derivation of Eq. (5)}

In this section, we provide the pedagogical derivation of Eq. (5). First, we recall the second Josephson relation:

$$
\frac{d \theta}{d t}=\frac{2 e}{\hbar} V,
$$

where $\theta$ is the overall phase of the condensate that represents the charge degree of freedom. Taking the spatial gradient yields

$$
\frac{d \mathbf{u}}{d t}=-\frac{2 e}{\hbar} \mathbf{E},
$$

where $\mathbf{u}=\boldsymbol{\nabla} \theta$ is the charge-phase distortion field. For the collection of charge vortices located at $\left\{\mathbf{r}_{i}(t)\right\}$, the distortion field can be written as

$$
\mathbf{u}\left(\mathbf{r} ;\left\{\mathbf{r}_{i}(t)\right\}\right)=-\boldsymbol{\nabla} \times\left[\hat{\mathbf{z}} \sum_{i} q_{i} \ln \left(\left|\mathbf{r}-\mathbf{r}_{i}(t)\right|\right)\right],
$$

where $q_{i}$ is the charge winding number of the $i$ th vortex, i.e., charge vorticity [65]. Taking the time derivative yields

$$
\begin{aligned}
\frac{d \mathbf{u}}{d t} & =\boldsymbol{\nabla} \times\left[\hat{\mathbf{z}} \sum_{i} q_{i} \mathbf{v}_{i} \cdot \nabla \ln \left(\left|\mathbf{r}-\mathbf{r}_{i}(t)\right|\right)\right] \\
& =-2 \pi \hat{\mathbf{z}} \times \sum_{i} q_{i} \mathbf{v}_{i} \delta\left(\mathbf{r}-\mathbf{r}_{i}\right) \\
& =-2 \pi \hat{\mathbf{z}} \times \mathbf{j}_{c},
\end{aligned}
$$

where $\mathbf{j}_{c}$ is the charge vortex current density. Plugging Eq. (20) into Eq. (18) gives

$$
\mathbf{E}=2 \pi \frac{\hbar}{2 e} \hat{\mathbf{z}} \times \mathbf{j}_{\mathrm{c}},
$$

which is Eq. (5) of the main text.

\section{B $\Delta V$ near the $d$-vector meron deconfinement}

Solving Eq. (10) for $T<T_{\mathrm{BKT}}^{\mathrm{dm}}$ with the boundary condition of Eq. (8) becomes simpler for the symmetric / antisymmetric setup $p_{l}= \pm p_{r}=p$ and $g_{l}=g_{r} \equiv g$ as it will give us the spin current $I^{\mathrm{sp}}$ that is an even / odd function of $x$. This enables us to obtain a first order differential equations for the spin current:

$$
\lambda \frac{d}{d x} \frac{I^{\mathrm{sp}}}{I_{0}^{\mathrm{sp}}}=\sqrt{\frac{\left|\frac{I^{\mathrm{sp}}}{I_{0}^{\mathrm{s}}}\right|^{2+2 T_{\mathrm{BKT}}^{\mathrm{dm}} / T}-\left|\frac{I^{\mathrm{sp}}(x=0)}{I_{0}^{\mathrm{sp}}}\right|^{2+2 T_{\mathrm{BKT}}^{\mathrm{dm}} / T}}{1+T_{\mathrm{BKT}}^{\mathrm{dm}} / T}},
$$

for the symmetric setup at $x>0$ and

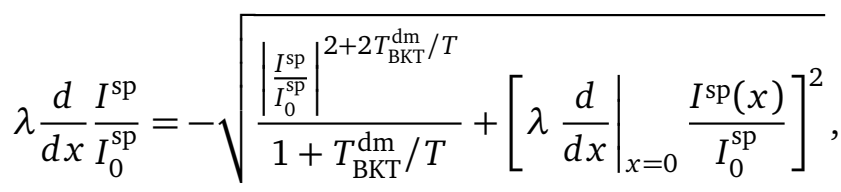

for the antisymmetric setup at $x>0$. 
For the symmetric setup below the transition, we obtain

$$
\begin{aligned}
\frac{d}{d x} I_{r}^{\mathrm{sp}}= & \frac{1}{\lambda} \frac{\hbar}{2 e} p I \frac{1}{\sqrt{1+T_{\mathrm{BKT}}^{\mathrm{dm}} / T}}\left|\frac{p \hbar I}{2 e I_{0}^{\mathrm{sp}}}\right|^{T_{\mathrm{BKT}}^{\mathrm{dm}} / T} \times \\
& \times \sqrt{1-C_{\text {even }}(T)\left(\frac{L / 2 \lambda}{\sqrt{1+\frac{T_{\mathrm{BKT}}^{\mathrm{dm}}}{T}}}\left|\frac{p \hbar I}{2 e I_{0}^{\mathrm{sp}}}\right|^{\frac{T_{\mathrm{BKT}}^{\mathrm{dm}}}{T}}+\frac{T}{T_{\mathrm{BKT}}^{\mathrm{dm}}}\right)^{-2-\frac{2 T}{T_{\mathrm{BKT}}^{\mathrm{dm}}}}},
\end{aligned}
$$

where $C_{\text {even }}(T)=\left[\sqrt{\pi} \frac{T}{T_{\mathrm{BKT}}^{\mathrm{dm}}} \frac{\Gamma\left(\frac{3+2 T / T_{\mathrm{BKT}}^{\mathrm{dm}}}{2+2 T / T_{\mathrm{BKT}}^{\mathrm{dm} T}}\right)}{\Gamma\left(\frac{2+T / T_{\mathrm{BT}}^{\mathrm{dm}}}{2+2 T / T_{\mathrm{BKT}}^{\mathrm{dm}}}\right)}\right]^{2+2 T / T_{\mathrm{BKT}}^{\mathrm{dm}}}$, by noting that Eq. (8) gives us $I_{r}^{\text {sp }} \approx p \hbar I / 2 e$ for $T>0$ in the $I \ll 2 e I_{0}^{\mathrm{sp}} / \hbar$ limit and inserting the relation between $I^{\mathrm{sp}}(x=0)$ and $I_{r}^{\mathrm{sp}}$ obtained from,

$$
\begin{aligned}
X=\int_{1}^{Y} \frac{d y}{\sqrt{y^{\alpha}-1}} & =\int_{1}^{\infty} \frac{d y}{\sqrt{y^{\alpha}-1}}-\int_{X}^{\infty} y^{-\frac{\alpha}{2}}\left(1+\frac{1}{2} y^{-\alpha}+\cdots\right) d y \\
& =\frac{\sqrt{\pi}}{\frac{\alpha}{2}-1} \frac{\Gamma(3 / 2-1 / \alpha)}{\Gamma(1-1 / \alpha)}-\frac{1}{\left(\frac{\alpha}{2}-1\right) Y^{\frac{\alpha}{2}-1}}+O\left(Y^{-\frac{3 \alpha}{2}+1}\right)
\end{aligned}
$$

back into Eq. (22). This gives us

$$
\begin{aligned}
\Delta V_{\text {even }} & =2 \frac{p}{g} \frac{2 e}{\hbar} \tilde{g} \frac{d}{d x} I_{r}^{\mathrm{sp}} \\
& =p^{2} \frac{2 I}{g} \frac{\tilde{g} / \lambda}{\sqrt{1+\frac{T_{\mathrm{BKT}}^{\mathrm{dm}}}{T}}}\left|\frac{p \hbar I}{2 e I_{0}^{\mathrm{sp}}}\right|^{\frac{T_{\mathrm{BKT}}^{\mathrm{dm}}}{T}}\left[1-\frac{1}{2} C_{\text {even }}(T)\left(\frac{L / 2 \lambda}{\left.\left.\sqrt{1+\frac{T_{\mathrm{BKT}}^{\mathrm{dm}}}{T}}\left|\frac{p \hbar I}{2 e I_{0}^{\mathrm{sp}}}\right|^{\frac{T_{\mathrm{BKT}}^{\mathrm{dm}}}{T}}\right)^{-2-\frac{2 T}{T_{\mathrm{BKT}}^{\mathrm{dm}}}}\right]}\right]\right.
\end{aligned}
$$

in the limit taken for Eq. (11).

For the antisymmetric setup below the transition, we obtain

$$
\begin{aligned}
\frac{d}{d x} I_{r}^{\mathrm{sp}}= & -\frac{1}{\lambda} \frac{\hbar}{2 e} p I \frac{1}{\sqrt{1+T_{\mathrm{BKT}}^{\mathrm{dm}} / T}}\left|\frac{p \hbar I}{2 e I_{0}^{\mathrm{sp}}}\right|^{T_{\mathrm{BKT}}^{\mathrm{dm}} / T} \times \\
& \times \sqrt{1+C_{o d d}(T)\left[\frac{L / 2 \lambda}{\left.\sqrt{1+\frac{T_{\mathrm{BKT}}^{\mathrm{dm}}}{T}}\left|\frac{p \hbar I}{2 e I_{0}^{\mathrm{sp}}}\right|^{\frac{T_{\mathrm{BK}}^{T}}{T}}+\frac{T}{T_{\mathrm{BKT}}^{\mathrm{dm}}}\right]^{-2-\frac{2 T}{T_{\mathrm{BKT}}^{\mathrm{dm}}}}}\right.},
\end{aligned}
$$

where $C_{\text {odd }}(T)=\left[\frac{\Gamma\left(\frac{T_{\mathrm{BK}}^{\mathrm{dm}} / T}{2+2 T_{\mathrm{BKT}}^{\mathrm{dm}} / T}\right) \Gamma\left(\frac{3+2 T_{\mathrm{BKT}}^{\mathrm{dm}} / T}{2+2 T_{\mathrm{BKT}}^{\mathrm{dm}} / T}\right)}{\sqrt{\pi\left(1+T_{\mathrm{BKT}}^{\mathrm{dm}} / T\right)}}\right]^{2}$, by noting that Eq. (8) gives us $I_{r}^{\mathrm{sp}} \approx-p \hbar I / 2 e$ for $T>0$ in the $I \ll 2 e I_{0}^{\mathrm{sp}} / \hbar$ limit and inserting the relation between $I^{\mathrm{sp}}(x=0)$ and $I_{r}^{\mathrm{sp}}$ obtained from,

$$
\begin{aligned}
X=\int_{0}^{Y} \frac{d y}{\sqrt{y^{\alpha}+1}} & =\int_{0}^{\infty} \frac{d y}{\sqrt{y^{\alpha}+1}}-\int_{Y}^{\infty} y^{-\frac{\alpha}{2}}\left(1-\frac{1}{2} y^{-\alpha}+\cdots\right) d y \\
& =\frac{\Gamma(1 / 2-1 / \alpha) \Gamma(1+1 / \alpha)}{\sqrt{\pi}}-\frac{1}{\left(\frac{\alpha}{2}-1\right) Y^{\frac{\alpha}{2}-1}}+O\left(Y^{-\frac{3 \alpha}{2}+1}\right),
\end{aligned}
$$


back into Eq. (23). This gives us

$$
\begin{aligned}
& \Delta V_{\text {odd }}=-2 \frac{p}{g} \frac{2 e}{\hbar} \tilde{g} \frac{d}{d x} I_{r}^{s p}
\end{aligned}
$$

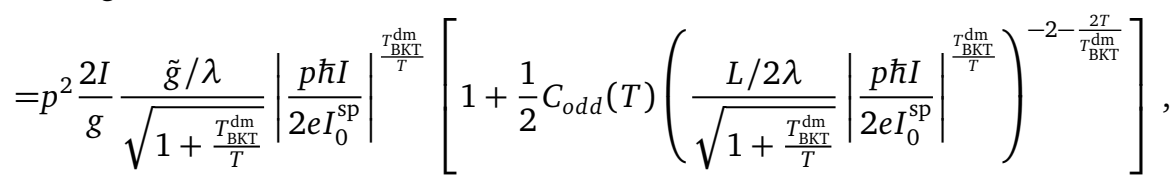

in the limit taken for Eq. (11). From Eqs. (25) and (27)

$$
V_{\mathrm{mr}}=\Delta V_{\text {even }}-\Delta V_{\text {odd }},
$$

gives us the magnetoresistance of Eq. (11).

For $T>T_{\mathrm{BKT}}^{\mathrm{dm}}$, the general solution of the now-linear Eq. (10),

$$
I^{\mathrm{sp}}(x)=\frac{1}{2}\left(I_{l}^{\mathrm{sp}}+I_{r}^{\mathrm{sp}}\right) \frac{\cosh \left(x / \lambda_{0}\right)}{\cosh \left(L / 2 \lambda_{0}\right)}-\frac{1}{2}\left(I_{l}^{\mathrm{sp}}-I_{r}^{\mathrm{sp}}\right) \frac{\sinh \left(x / \lambda_{0}\right)}{\sinh \left(L / 2 \lambda_{0}\right)},
$$

can be inserted into Eq. (8) to give us, up to the first order in $e^{-L / \lambda_{0}}$,

$$
\begin{aligned}
& \frac{d}{d x} I_{l}^{\mathrm{sp}}=-\frac{1}{\lambda_{0}}\left(I_{l}^{\mathrm{sp}}-2 I_{r}^{\mathrm{sp}} e^{-L / \lambda_{0}}\right)=-\frac{\hbar}{2 e} \frac{I}{\lambda_{0}} \frac{p_{l}\left[1+\left(1-p_{r}^{2}\right) \frac{\tilde{g}_{r}}{\lambda_{0}}\right]-2 p_{r} e^{-L / \lambda_{0}}}{\left[1+\left(1-p_{l}^{2}\right) \frac{\tilde{g}_{l}}{\lambda_{0}}\right]\left[1+\left(1-p_{r}^{2}\right) \frac{\tilde{g}_{r}}{\lambda_{0}}\right]}, \\
& \frac{d}{d x} I_{r}^{\mathrm{sp}}=\frac{1}{\lambda_{0}}\left(I_{r}^{\mathrm{sp}}-2 I_{l}^{\mathrm{sp}} e^{-L / \lambda_{0}}\right)=\frac{\hbar}{2 e} \frac{I}{\lambda_{0}} \frac{p_{r}\left[1+\left(1-p_{l}^{2}\right) \frac{\tilde{g}_{l}}{\lambda_{0}}\right]-2 p_{l} e^{-L / \lambda_{0}}}{\left[1+\left(1-p_{l}^{2}\right) \frac{\tilde{g}_{l}}{\lambda_{0}}\right]\left[1+\left(1-p_{r}^{2}\right) \frac{\tilde{g}_{r}}{\lambda_{0}}\right]} .
\end{aligned}
$$

From these boundary values of $d I^{\mathrm{sp}} / d x$ and from the relation,

$$
\Delta V\left(p_{l}, p_{r}\right)=-\frac{2 e}{\hbar}\left(\frac{p_{l}}{g_{l}} \tilde{g}_{l} \frac{d}{d x} I_{l}^{\mathrm{sp}}-\frac{p_{r}}{g_{r}} \tilde{g}_{r} \frac{d}{d x} I_{r}^{\mathrm{sp}}\right),
$$

we obtain

$$
\Delta V\left(p_{l}, p_{r}\right)=\frac{I}{g_{l}+g_{r}} \frac{\tilde{g}_{l}+\tilde{g}_{r}}{\lambda_{0}} \frac{p_{l}^{2}\left[1+\left(1-p_{r}^{2}\right) \frac{\tilde{g}_{r}}{\lambda_{0}}\right]+p_{r}^{2}\left[1+\left(1-p_{l}^{2}\right) \frac{\tilde{g}_{l}}{\lambda_{0}}\right]-4 p_{l} p_{r} e^{-L / \lambda_{0}}}{\left[1+\left(1-p_{l}^{2}\right) \frac{\tilde{g}_{l}}{\lambda_{0}}\right]\left[1+\left(1-p_{r}^{2}\right) \frac{\tilde{g}_{r}}{\lambda_{0}}\right]},
$$

up to the first order in $e^{-L / \lambda_{0}}$, from which $V_{\mathrm{mr}} \equiv \Delta V\left(p_{l}=-p_{r}=p\right)-\Delta V\left(p_{l}=p_{r}=p\right)$ can be obtained.

\section{C $\quad \Delta V$ near the half-quantum vortex deconfinement}

In terms of transport, the defining characteristics of hqv's is that they contribute to both the charge and the spin terms to $\Delta V$,

$$
\Delta V=\int_{-L / 2}^{L / 2} d x j_{c}-\frac{2 e}{\hbar}\left(\frac{p_{l}}{g_{l}} \tilde{g}_{l} \frac{d}{d x} I_{l}^{\mathrm{sp}}-\frac{p_{r}}{g_{r}} \tilde{g}_{r} \frac{d}{d x} I_{r}^{\mathrm{sp}}\right) .
$$

The results presented in the main text shows that for any finite temperature the spin term should be in the same form as $\Delta V$ of Eq. (29). The charge term also differs from that of fqv's as the spin vorticity current is not uniform for $T<T_{\mathrm{BKT}}^{\mathrm{hqv}}$ :

$$
j_{c} \propto \frac{1}{2} \sum_{\sigma}\left(I+\sigma \frac{2 e}{\hbar} I^{\mathrm{sp}}\right)\left|I+\sigma \frac{2 e}{\hbar} I^{\mathrm{sp}}\right|^{\frac{2 T_{\mathrm{BKT}}^{\mathrm{hqv}}}{T}} \approx I|I|^{\frac{2 T_{\mathrm{BKT}}^{\mathrm{hqv}}}{T}}\left[1+\frac{T_{\mathrm{BKT}}^{\mathrm{hqv}}}{T}\left(\frac{2 T_{\mathrm{BKT}}^{\mathrm{hqv}}}{T}+1\right)\left|\frac{2 e}{\hbar} \frac{I^{\mathrm{sp}}}{I}\right|^{2}\right] .
$$


Altogether we obtain up to the first order in $e^{-L / \tilde{\lambda}_{\text {eff }}}$

$$
\begin{aligned}
& \Delta V\left(p_{l}, p_{r}\right)=\frac{L}{w} \tilde{\rho}_{0}^{\mathrm{hqv}} I\left|\frac{I}{I_{0}^{\mathrm{hqv}}}\right|^{2 T_{\mathrm{BKT}}^{\mathrm{fqv}} / T}\left[1+\frac{\tilde{\lambda}_{\text {eff }}}{2 L} \frac{T_{\mathrm{BKT}}^{\mathrm{hqv}}}{T}\left(\frac{2 T_{\mathrm{BKT}}^{\mathrm{hqv}}}{T}+1\right) C\left(p_{l}, p_{r}\right)\right] \\
& +\frac{I}{g_{l}+g_{r}} \frac{\tilde{g}_{l}+\tilde{g}_{r}}{\tilde{\lambda}_{\text {eff }}} \frac{p_{l}^{2}\left[1+\left(1-p_{r}^{2}\right) \frac{\tilde{g}_{r}}{\hat{\mathrm{e}}_{\mathrm{eff}}}\right]+p_{r}^{2}\left[1+\left(1-p_{l}^{2}\right) \frac{\tilde{g}_{l}}{\tilde{\hat{\lambda}}_{\mathrm{eff}}}\right]-4 p_{l} p_{r} e^{-L / \tilde{\lambda}_{\text {eff }}}}{\left[1+\left(1-p_{l}^{2}\right) \frac{\tilde{g}_{l}}{\tilde{\lambda}_{\text {eff }}}\right]\left[1+\left(1-p_{r}^{2}\right) \frac{\tilde{g}_{r}}{\tilde{\lambda}_{\text {eff }}}\right]},
\end{aligned}
$$

where $\tilde{\rho}_{0}^{\text {hqv }}$ is a phenomenological constant in the units of 2D resistivity and the constant

$$
\begin{aligned}
C\left(p_{l}, p_{r}\right)= & \frac{p_{l}^{2}\left[1+\left(1-p_{r}^{2}\right) \frac{\tilde{g}_{r}}{\tilde{\lambda}_{\text {eff }}}\right]^{2}+p_{r}^{2}\left[1+\left(1-p_{l}^{2}\right) \frac{\tilde{g}_{l}}{\tilde{\lambda}_{\text {eff }}}\right]^{2}}{\left[1+\left(1-p_{l}^{2}\right) \frac{\tilde{g}_{l}}{\hat{\lambda}_{\text {eff }}}\right]^{2}\left[1+\left(1-p_{r}^{2}\right) \frac{\tilde{s}_{r}}{\hat{\lambda}_{\text {eff }}}\right]^{2}} \\
& -\frac{2 p_{l} p_{r}\left[2+\left(1-p_{l}^{2}\right) \frac{\tilde{g}_{l}}{\tilde{\lambda}_{\text {eff }}}+\left(1-p_{r}^{2}\right) \frac{\tilde{g}_{r}}{\tilde{\lambda}_{\text {eff }}}\right] e^{-L / \tilde{\lambda}_{\text {eff }}}}{\left[1+\left(1-p_{l}^{2}\right) \frac{\tilde{g}_{l}}{\tilde{\lambda}_{\text {eff }}}\right]^{2}\left[1+\left(1-p_{r}^{2}\right) \frac{\tilde{g}_{r}}{\tilde{\lambda}_{\text {eff }}}\right]^{2}} \\
& +\frac{4 L}{\tilde{\lambda}_{\text {eff }}} \frac{p_{r} e^{-L / \tilde{\lambda}_{\text {eff }}}}{\left[1+\left(1-p_{l}^{2}\right) \frac{\tilde{g}_{l}}{\tilde{\lambda}_{\text {eff }}}\right]\left[1+\left(1-p_{r}^{2}\right) \frac{\tilde{g}_{r}}{\tilde{\lambda}_{\text {eff }}}\right]}
\end{aligned}
$$

that vanishes when $p_{l}=p_{r}=0$. From this, we obtain the magnetoresistance of Eq. (15) by taking the limit where both $L \gg \tilde{\lambda}_{\text {eff }}$ and $I \ll I_{0}^{\text {hqv }}$ are satisfied.

The charge vorticity current is uniform for $T>T_{\mathrm{BKT}}^{\mathrm{hqv}}$, in which case

$$
\begin{aligned}
\Delta V= & \frac{L}{w}\left(\frac{\pi \hbar}{e}\right)^{2} \mu_{\mathrm{hqv}} n_{f} I \\
& \left.+\frac{I}{g_{l}+g_{r}} \frac{\tilde{g}_{l}+\tilde{g}_{r}}{\tilde{\lambda}_{0}} \frac{p_{l}^{2}\left[1+\left(1-p_{r}^{2}\right) \frac{\tilde{g}_{r}}{\tilde{\lambda}_{0}}\right]+p_{r}^{2}\left[1+\left(1-p_{l}^{2}\right) \frac{\tilde{g}_{l}}{\tilde{\lambda}_{0}}\right]-4 p_{l} p_{r} e^{-L / \tilde{\lambda}_{0}}}{\left[1+\left(1-p_{l}^{2}\right) \frac{\tilde{g}_{l}}{\tilde{\lambda}_{0}}\right]\left[1+\left(1-p_{r}^{2}\right) \tilde{\bar{\lambda}}_{0}\right.}\right]
\end{aligned}
$$

easily reduces to Eq. (16).

\section{References}

[1] A. J. Leggett, A theoretical description of the new phases of liquid ${ }^{3} \mathrm{He}$, Rev. Mod. Phys. 47, 331 (1975), doi:10.1103/RevModPhys.47.331.

[2] M. Sigrist and K. Ueda, Phenomenological theory of unconventional superconductivity, Rev. Mod. Phys. 63, 239 (1991), doi:10.1103/RevModPhys.63.239.

[3] A. P. Mackenzie and Y. Maeno, The superconductivity of $\mathrm{sr}_{2} \mathrm{ruO}_{4}$ and the physics of spintriplet pairing, Rev. Mod. Phys. 75, 657 (2003), doi:10.1103/RevModPhys.75.657.

[4] D. Vollhardt and P. Woelfle, The superfluid phases of Helium 3, Taylor \& Francis, ISBN 9780850664126, (1990).

[5] S. S. Saxena et al., Superconductivity on the border of itinerant-electron ferromagnetism in $\mathrm{UGe}_{2}$, Nature 406, 587 (2000), doi:10.1038/35020500. 
[6] D. Aoki, A. Huxley, E. Ressouche, D. Braithwaite, J. Flouquet, J.-P. Brison, E. Lhotel and C. Paulsen, Coexistence of superconductivity and ferromagnetism in URhGe, Nature 413, 613 (2001), doi:10.1038/35098048.

[7] N. T. Huy et al., Superconductivity on the border of weak itinerant ferromagnetism in UCoGe, Phys. Rev. Lett. 99, 067006 (2007), doi:10.1103/PhysRevLett.99.067006.

[8] S. Ran et al., Nearly ferromagnetic spin-triplet superconductivity, Science 365, 684 (2019), doi:10.1126/science.aav8645.

[9] L. Jiao, S. Howard, S. Ran, Z. Wang, J. Olivares Rodriguez, M. Sigrist, Z. Wang, N. P. Butch and V. Madhavan, Chiral superconductivity in heavy-fermion metal UTe $e_{2}$, Nature 579, 523 (2020), doi:10.1038/s41586-020-2122-2.

[10] L. Fu and E. Berg, Odd-parity topological superconductors: Theory and application to $\mathrm{Cu}_{x} \mathrm{bi}_{2} \mathrm{Se}_{3}$, Phys. Rev. Lett. 105, 097001 (2010), doi:10.1103/PhysRevLett.105.097001.

[11] K. Matano, M. Kriener, K. Segawa, Y. Ando and G.-q. Zheng, Spin-rotation symmetry breaking in the superconducting state of $\mathrm{Cu}_{x} \mathrm{Bi}_{2} \mathrm{Se}_{3}$, Nat. Phys. 12, 852 (2016), doi:10.1038/nphys3781.

[12] L. P. Gor'kov and E. I. Rashba, Superconducting $2 D$ system with lifted spin degeneracy: Mixed singlet-triplet state, Phys. Rev. Lett. 87, 037004 (2001), doi:10.1103/PhysRevLett.87.037004.

[13] C. R. Reeg and D. L. Maslov, Proximity-induced triplet superconductivity in Rashba materials, Phys. Rev. B 92, 134512 (2015), doi:10.1103/PhysRevB.92.134512.

[14] J. Cayao and A. M. Black-Schaffer, Odd-frequency superconducting pairing in junctions with Rashba spin-orbit coupling, Phys. Rev. B 98, 075425 (2018), doi:10.1103/PhysRevB.98.075425.

[15] C. Xu and L. Balents, Topological superconductivity in twisted multilayer graphene, Phys. Rev. Lett. 121, 087001 (2018), doi:10.1103/PhysRevLett.121.087001.

[16] Y.-Z. You and A. Vishwanath, Superconductivity from valley fluctuations and approximate SO(4) symmetry in a weak coupling theory of twisted bilayer graphene, npj Quantum Mater. 4, 16 (2019), doi:10.1038/s41535-019-0153-4.

[17] B. Lian, Z. Wang and B. Andrei Bernevig, Twisted bilayer graphene: A phonon-driven superconductor, Phys. Rev. Lett. 122, 257002 (2019), doi:10.1103/PhysRevLett.122.257002.

[18] J. Y. Lee, E. Khalaf, S. Liu, X. Liu, Z. Hao, P. Kim and A. Vishwanath, Theory of correlated insulating behaviour and spin-triplet superconductivity in twisted double bilayer graphene, Nat. Commun. 10, 5333 (2019), doi:10.1038/s41467-019-12981-1.

[19] M. S. Scheurer and R. Samajdar, Pairing in graphene-based Moiré superlattices, Phys. Rev. Research 2, 033062 (2020), doi:10.1103/PhysRevResearch.2.033062.

[20] Y. Wang, J. Kang and R. M. Fernandes, Topological and nematic superconductivity mediated by ferro-SU(4) fluctuations in twisted bilayer graphene, Phys. Rev. B 103, 024506 (2021), doi:10.1103/PhysRevB.103.024506.

[21] T. M. Rice and M. Sigrist, $\mathrm{Sr}_{2} \mathrm{RuO}_{4}$ : An electronic analogue of ${ }^{3} \mathrm{He}$ ?, J. Phys.: Condens. Matter 7, L643 (1995), doi:10.1088/0953-8984/7/47/002. 
[22] A. Pustogow et al., Constraints on the superconducting order parameter in $\mathrm{Sr}_{2} \mathrm{RuO}_{4}$ from oxygen-17 nuclear magnetic resonance, Nature 574, 72 (2019), doi:10.1038/s41586-019$1596-2$.

[23] A. Borovik-Romanov, Y. M. Bunkov, V. Dmitriev and Y. M. Mukharskiy, Long-lived induction signal in superfluid ${ }^{3} \mathrm{He}-\mathrm{B}$, JETP Lett. 40, 1033 (1984).

[24] I. Fomin, Long-lived induction signal and spatially nonuniform spin precession in ${ }^{3} \mathrm{He}-\mathrm{B}$, JETP Lett. 40, 1037 (1984).

[25] S. Bum Chung, S. Kwon Kim, K. Hoon Lee and Y. Tserkovnyak, Cooper-pair spin current in a strontium ruthenate heterostructure, Phys. Rev. Lett. 121, 167001 (2018), doi:10.1103/PhysRevLett.121.167001.

[26] E. B. Sonin, Analogs of superfluid currents for spins and electron-hole pairs, Sov. Phys. JETP 47, 1091 (1978).

[27] E. B. Sonin, Spin currents and spin superfluidity, Adv. Phys. 59, 181 (2010), doi:10.1080/00018731003739943.

[28] V. L. Berezinskii, Destruction of long-range order in one-dimensional and two-dimensional dystems having a continuous symmetry group: I. Classical systems, Sov. Phys. JETP 32, 493 (1971).

[29] J. M. Kosterlitz and D. J. Thouless, Ordering, metastability and phase transitions in twodimensional systems, J. Phys. C: Solid State Phys. 6, 1181 (1973), doi:10.1088/0022$3719 / 6 / 7 / 010$.

[30] B. I. Halperin, G. Refael and E. Demler, Resistance in superconductors, Int. J. Mod. Phys. B 24, 4039 (2010), doi:10.1142/s021797921005644x.

[31] J. Linder and J. W. A. Robinson, Superconducting spintronics, Nat. Phys. 11, 307 (2015), doi:10.1038/nphys3242.

[32] S. Kwon Kim and S. Bum Chung, Transport signature of the magnetic Berezinskii-KosterlitzThouless transition, SciPost Phys. 10, 068 (2021), doi:10.21468/SciPostPhys.10.3.068.

[33] S. Takei and Y. Tserkovnyak, Superfluid spin transport through easy-plane ferromagnetic insulators, Phys. Rev. Lett. 112, 227201 (2014), doi:10.1103/PhysRevLett.112.227201.

[34] G. E. Volovik and V. P. Mineev, Vortices with free ends in superfluid he/sup 3/-a, JETP Lett. 24, 561 (1976).

[35] S. Bum Chung, H. Bluhm and E.-A. Kim, Stability of half-quantum vortices $p_{x}+i p_{y}$ superconductors, Phys. Rev. Lett. 99, 197002 (2007), doi:10.1103/PhysRevLett.99.197002.

[36] J. Jang, D. G. Ferguson, V. Vakaryuk, R. Budakian, S. B. Chung, P. M. Goldbart and Y. Maeno, Observation of Half-Height Magnetization Steps in $\mathrm{Sr}_{2} \mathrm{RuO}_{4}$, Science 331, 186 (2011), doi:10.1126/science.1193839.

[37] S. Autti, V. V. Dmitriev, J. T. Mäkinen, A. A. Soldatov, G. E. Volovik, A. N. Yudin, V. V. Zavjalov and V. B. Eltsov, Observation of half-quantum vortices in topological superfluid ${ }^{3}$ He, Phys. Rev. Lett. 117, 255301 (2016), doi:10.1103/PhysRevLett.117.255301.

[38] X. Cai, B. M. Zakrzewski, Y. A. Ying, H.-Y. Kee, M. Sigrist, J. Elliott Ortmann, W. Sun, Z. Mao and Y. Liu, Magnetoresistance oscillation study of the half-quantum vortex in doubly connected mesoscopic superconducting cylinders of $\mathrm{Sr}_{2} \mathrm{RuO}_{4}$, arXiv:2010.15800. 
[39] E. Babaev, Phase diagram of planar $U(1) \times U(1)$ superconductor, Nucl. Phys. B 686, 397 (2004), doi:10.1016/j.nuclphysb.2004.02.021.

[40] D. F. Agterberg and H. Tsunetsugu, Dislocations and vortices in pair-density-wave superconductors, Nat. Phys. 4, 639 (2008), doi:10.1038/nphys999.

[41] E. Berg, E. Fradkin and S. A. Kivelson, Charge-4e superconductivity from pair-densitywave order in certain high-temperature superconductors, Nat. Phys. 5, 830 (2009), doi:10.1038/nphys1389.

[42] D. F. Agterberg, M. Sigrist and H. Tsunetsugu, Order parameter and vortices in the superconducting $q$ phase of cecoin $_{5}$, Phys. Rev. Lett. 102, 207004 (2009), doi:10.1103/PhysRevLett.102.207004.

[43] L. Radzihovsky and A. Vishwanath, Quantum liquid crystals in an imbalanced Fermi gas: Fluctuations and fractional vortices in Larkin-Ovchinnikov states, Phys. Rev. Lett. 103, 010404 (2009), doi:10.1103/PhysRevLett.103.010404.

[44] Y. Wang, G. Young Cho, T. L. Hughes and E. Fradkin, Topological superconducting phases from inversion symmetry breaking order in spin-orbit-coupled systems, Phys. Rev. B 93, 134512 (2016), doi:10.1103/PhysRevB.93.134512.

[45] M. E. Peskin, Mandelstam-'t Hooft duality in Abelian lattice models, Ann. Phys. 113, 122 (1978), doi:10.1016/0003-4916(78)90252-X.

[46] P. Minnhagen, The two-dimensional Coulomb gas, vortex unbinding, and superfluid-superconducting films, Rev. Mod. Phys. 59, 1001 (1987), doi:10.1103/RevModPhys.59.1001.

[47] M. P. A. Fisher and D. H. Lee, Correspondence between two-dimensional bosons and a bulk superconductor in a magnetic field, Phys. Rev. B 39, 2756 (1989), doi:10.1103/PhysRevB.39.2756.

[48] Y. Tserkovnyak and J. Zou, Quantum hydrodynamics of vorticity, Phys. Rev. Research 1, 033071 (2019), doi:10.1103/PhysRevResearch.1.033071.

[49] S. Dasgupta, S. Zhang, I. Bah and O. Tchernyshyov, Quantum statistics of vortices from a dual theory of the XY ferromagnet, Phys. Rev. Lett. 124, 157203 (2020), doi:10.1103/PhysRevLett.124.157203.

[50] D. Podolsky, S. Chandrasekharan and A. Vishwanath, Phase transitions of $s=1$ spinor condensates in an optical lattice, Phys. Rev. B 80, 214513 (2009), doi:10.1103/PhysRevB.80.214513.

[51] D. A. Ivanov, Non-Abelian statistics of half-quantum vortices in p-wave superconductors, Phys. Rev. Lett. 86, 268 (2001), doi:10.1103/PhysRevLett.86.268.

[52] D. Ariad, E. Grosfeld and B. Seradjeh, Effective theory of vortices in twodimensional spinless chiral p-wave superfluids, Phys. Rev. B 92, 035136 (2015), doi:10.1103/PhysRevB.92.035136.

[53] B. I. Halperin and D. R. Nelson, Resistive transition in superconducting films, J. Low. Temp. Phys. 36, 599 (1979), doi:10.1007/BF00116988.

[54] F. Krüger and S. Scheidl, Nonuniversal ordering of spin and charge in stripe phases, Phys. Rev. Lett. 89, 095701 (2002), doi:10.1103/PhysRevLett.89.095701. 
[55] A. Vargunin and M. Silaev, Flux flow spin Hall effect in type-II superconductors with spinsplitting field, Sci. Rep. 9, 5914 (2019), doi:10.1038/s41598-019-42034-y.

[56] N. P. Breznay, M. A. Steiner, S. Allan Kivelson and A. Kapitulnik, Self-duality and a Hallinsulator phase near the superconductor-to-insulator transition in indium-oxide films, Proc. Natl. Acad. Sci. 113, 280 (2015), doi:10.1073/pnas.1522435113.

[57] F. Meier and D. Loss, Magnetization transport and quantized spin conductance, Phys. Rev. Lett. 90, 167204 (2003), doi:10.1103/PhysRevLett.90.167204.

[58] K. Aoyama and H. Kawamura, Effects of magnetic anisotropy on spin and thermal transport in classical antiferromagnets on the square lattice, Phys. Rev. B 100, 144416 (2019), doi:10.1103/PhysRevB.100.144416.

[59] M. S. Anwar et al., Direct penetration of spin-triplet superconductivity into a ferromagnet in $\mathrm{Au} / \mathrm{SrRuO}_{3} / \mathrm{Sr}_{2} \mathrm{RuO}_{4}$ junctions, Nat. Commun. 7, 13220 (2016), doi:10.1038/ncomms13220.

[60] A. M. Kadin, K. Epstein and A. M. Goldman, Renormalization and the KosterlitzThouless transition in a two-dimensional superconductor, Phys. Rev. B 27, 6691 (1983), doi:10.1103/PhysRevB.27.6691.

[61] A. T. Fiory, A. F. Hebard and W. I. Glaberson, Superconducting phase transitions in indium/indium-oxide thin-film composites, Phys. Rev. B 28, 5075 (1983), doi:10.1103/PhysRevB.28.5075.

[62] A. F. Hebard and A. T. Fiory, Critical-exponent measurements of a two-dimensional superconductor, Phys. Rev. Lett. 50, 1603 (1983), doi:10.1103/PhysRevLett.50.1603.

[63] E. Babaev, Fractional-flux vortices and spin superfluidity in triplet superconductors, Phys. Rev. Lett. 94, 137001 (2005), doi:10.1103/PhysRevLett.94.137001.

[64] J. V. José, L. P. Kadanoff, S. Kirkpatrick and D. R. Nelson, Renormalization, vortices, and symmetry-breaking perturbations in the two-dimensional planar model, Phys. Rev. B 16, 1217 (1977), doi:10.1103/PhysRevB.16.1217.

[65] M. Kardar, Statistical physics of fields, Cambridge University Press, Cambridge, UK, ISBN 9780511815881 (2007), doi:10.1017/СBO9780511815881. 